\title{
Tracheobronchopathia osteochondroplastica - a rare cause of recurrent pneumonia
}

\author{
Undugodage U C M ${ }^{1}$, Punchihewa $R^{2}$, Gunasinghe $\mathbf{W}^{3}$, Gunasekera K D G
}

Journal of the Ceylon College of Physicians, 2017, 48, 82-84

\section{Case report}

We report a case of a 55-year-old male who presented with fever and cough for 10 days. He had a low-grade fever and his cough was productive with one episode of haemoptysis. His past records showed that he has had several episodes of right middle lobe pneumonia within the last 4 months. On examination, he had coarse crepitations over the right axilla. There was no lymphadenopathy.

He has had well controlled type 2 DM for 4 years and hypertension for 15 years. He was a non-smoker. Investigations revealed a WBC of $16,000 / \mathrm{mm}^{3}$ with $80 \%$ neutrophils with a CRP of $85 \mathrm{mg} / \mathrm{dL}$. Chest X-ray showed a right middle lobe consolidation, similar to previous X-rays. His renal function tests and the liver function tests were normal. He was investigated for recurrent right middle lobe pneumonia. Sputum for Acid Fast Bacilli (AFB) and Mantoux tests were negative. Retroviral screen was negative.

In view of the possible underlying malignancy occluding the right middle lobe bronchus we proceeded with a fiberoptic bronchoscopy (FOB) and a contrast enhanced $\mathrm{CT}$ chest. FOB showed extensive involvement of the trachea with multiple nodules projecting into the lumen. (Figure 2,3). These extended into the right main bronchus. The nodules were the same colour as the bronchial mucosa. A tentative diagnosis of malignancy infiltrating the trachea and the bronchial tree was made. We also noted that the biopsy of these nodules was difficult as they were hard in consistency.

Contrast enhanced CT chest, was reported as a consolidation in the right middle lobe, without any other abnormality.

Histology showed a bronchial mucosa with nodular deposition of sub epithelial cartilage and bony tissues. Formation of bony tissue with marrow spaces was noted in the submucosa. Overlying respiratory epithelium was normal (Figure 4,5). A diagnosis of tracheobronchopathia osteochondroplastica (TPO) was made based on these findings.

A closer review of the CT scan showed nodularity was on the anterior wall of the trachea, with sparing of the posterior tracheal wall (Figure 1).

A repeat bronchoscopy was done and we noted that although the trachea was extensively involved the posterior tracheal wall was free of the nodules.

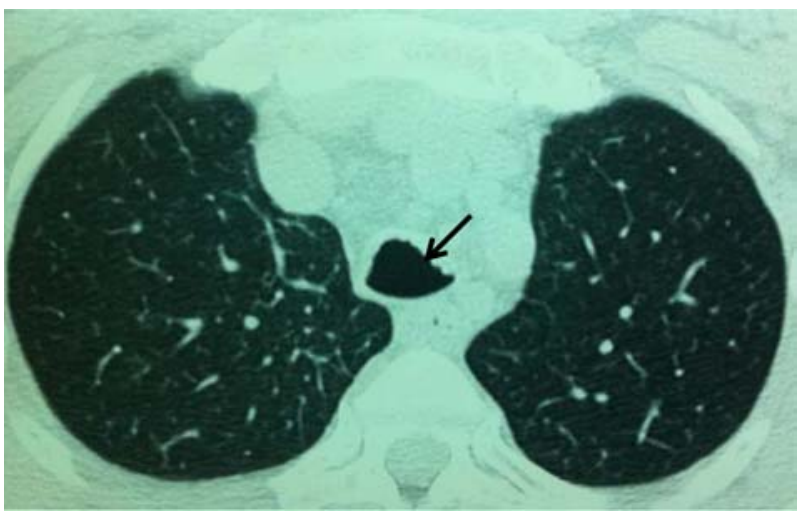

Figure 1. CT scan of the chest - arrow showing the nodularity on the posterior wall of the trachea.

\footnotetext{
${ }^{1}$ Department of Physiology, Faculty of Medical Sciences, University of Sri Jayawardenapura, Sri Lanka.

${ }^{2}$ National Hospital for Respiratory Diseases, Welisara, Sri Lanka.

${ }^{3}$ District General Hospital, Monaragala, Sri Lanka.

${ }^{4}$ National Hospital Sri Lanka, Central Chest Clinic, Sri Lanka.
}

Corresponding author: Undugodage UCM

Email: chandimanin@yahoo.com 

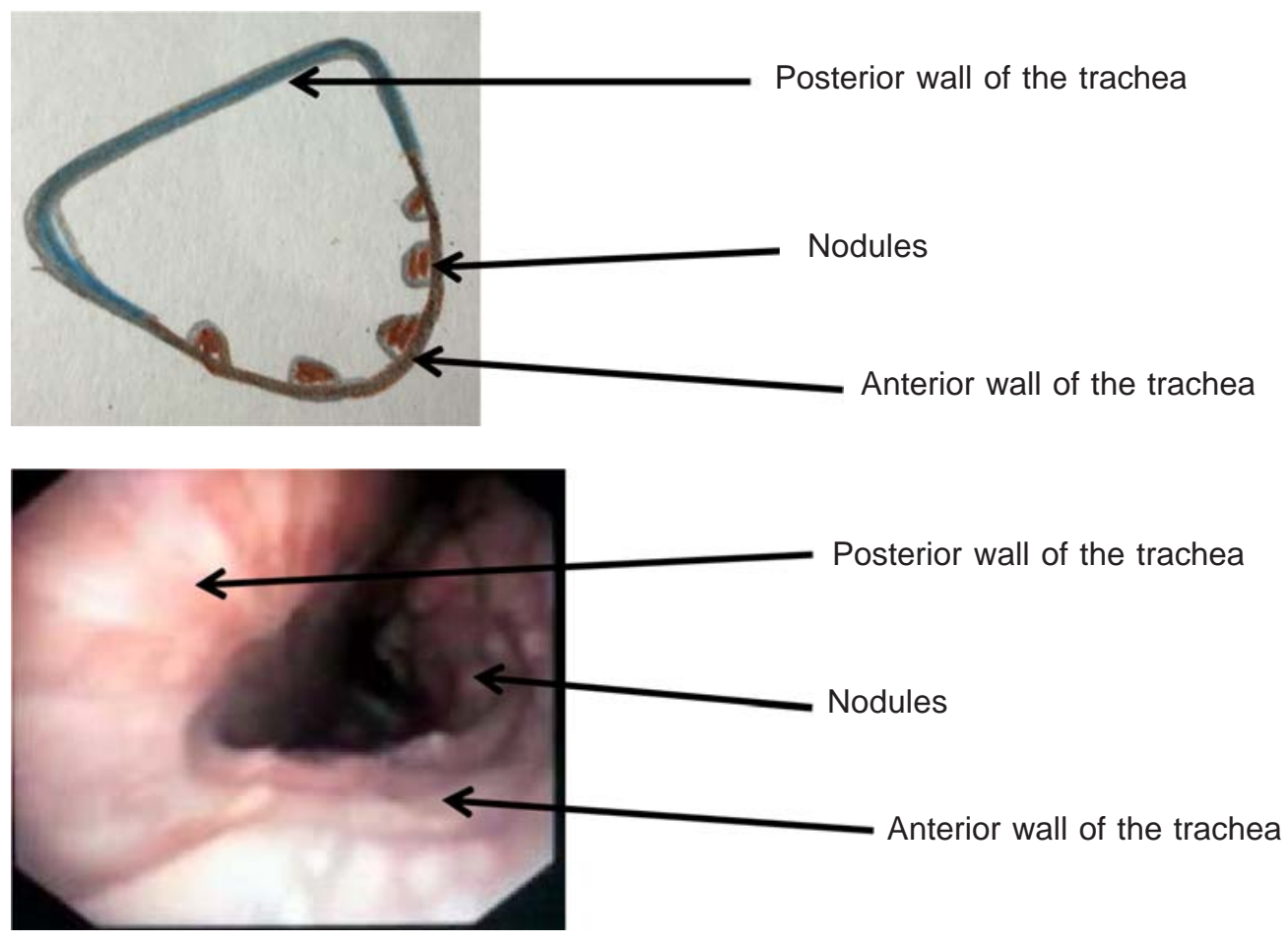

Figure 2. Line diagram of a cross section of the trachea (upper) Bronchoscopic view of the trachea (lower).

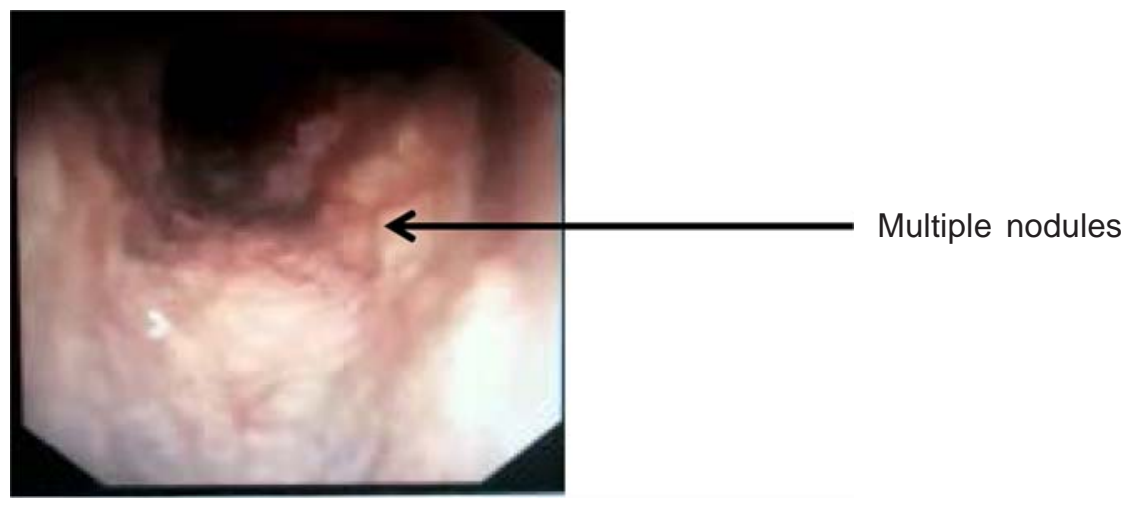

Figure 3. Bronchoscopic view of the trachea.

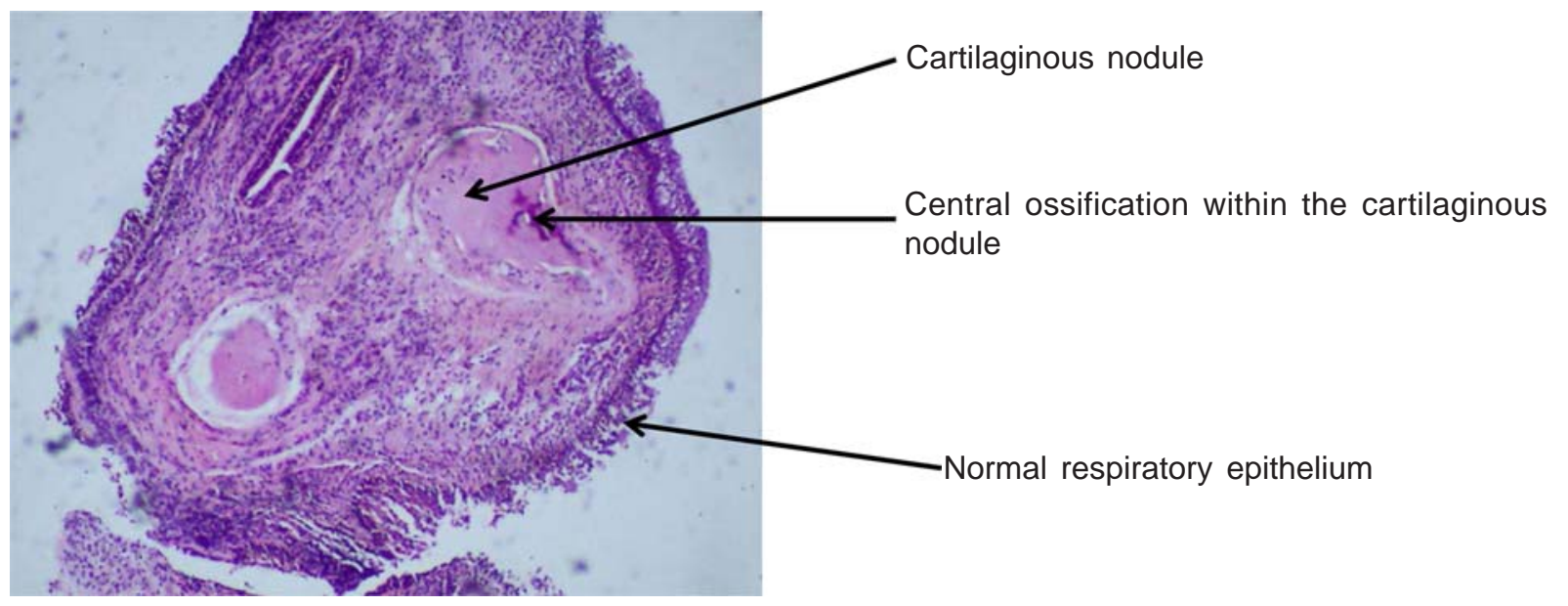

Figure 4. Sub mucosal cartilaginous nodules with ossification. The overlying respiratory epithelium is intact and normal. 


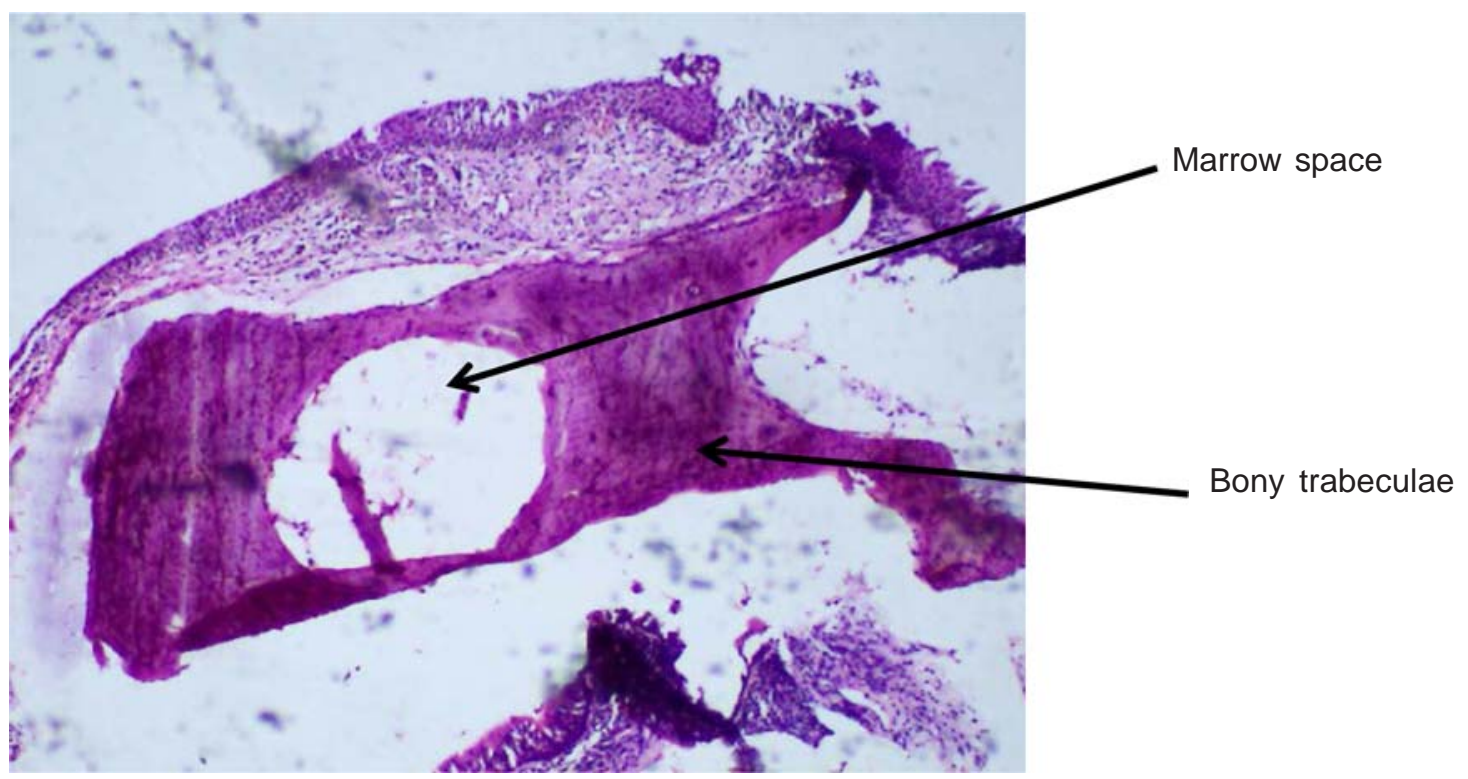

Figure 5. Submucosal bony tissue with marrow spaces.

\section{Discussion}

TPO is a rare benign disease of unknown etiology characterized by multiple cartilaginous or bony submucosal nodules, which project into the tracheobronchial lumen ${ }^{1}$. The nodules originate in the airway cartilages and thus typically spare the posterior membranous wall of the trachea.

Both the incidence and prevalence of the disorder in the general population are unknown. The incidence of TPO in a large series of 16888 bronchoscopies was 1:1299 bronchoscopies ${ }^{2}$.

These patients' present with cough, wheezing and recurrent respiratory tract infections. The symptoms are due to narrowing and thickening of airways.

The bronchoscopic appearance alone is diagnostic of the disease, however biopsy of the airway lesions is conclusive. In severe cases CT scan may reveal bony spicules in the trachea ${ }^{3}$. Histology reveals bone formation within the sub mucosa with normal overlying respiratory epithelium. Some bony tissue can form marrow spaces.

The differential diagnosis includes tracheobronchial amyloidosis, calcified lesions secondary to TB, carcinoma, papilloma, fibroma, endobronchial sarcoidosis, polychondritis, and Wegener's granulomatosis. These can be excluded on histology.
Treatment is only symptomatic, which includes antibiotics in case of bacterial infections. Mechanical measures such as stenting can be used to remove obstruction of nodules if symptomatic.

The disease usually remains stable for years or progresses very slowly ${ }^{4}$.

However, progression has been reported eventually leading to respiratory insufficiency ${ }^{5}$. Only a minority of cases develops significant upper airway obstruction and require invasive procedures on affected airways.

\section{References}

1. Luo S, Wu L, Zhou J, Xu S, Yang Q, Li Y, Shen H, Zhang S. Tracheobronchopathia osteochondroplastica: two cases and a review of the literature. Int J Clin Exp Pathol. 2015; 8(7): 8585-90. eCollection 2015.

2. Prakash UB. Tracheobronchopathia osteochondroplastica. Semin Respir Crit Care Med 2002, 23: 167-75.

3. Restrepo S, Pandit M, Villamil MA, Rojas IC, Perez JM, Gascue A: Tracheobronchopathia osteochondroplastica: helical CT findings in 4 cases. J Thorac Imaging 2004; 19: 112-16.

4. Decalmer S, Woodcock A, Greaves M, Howe M, Smith J. Airway abnormalities at flexible bronchoscopy in patients with chronic cough. Eur Respir J 2007, 30: 1138-42.

5. Molloy AR, McMahon JN. Rapid progression of tracheal stenosis associated with tracheopathia osteochondroplastica. Intensive Care Med 1988, 15: 60-2. 